\title{
Quasiparticle picture of quarks near chiral transition at finite temperature
}

\author{
M. Kitazawa ${ }^{\mathrm{a}}$, T. Kunihiro ${ }^{\mathrm{b}}$, and Y. Nemoto $^{\mathrm{c}}$ \\ a RIKEN BNL Research Center, Brookhaven National Laboratory, Upton, NY 11973, \\ USA \\ bYukawa Institute for Theoretical Physics, Kyoto University, Kyoto, 606-8502, Japan \\ cDepartment of Physics, Nagoya University, Nagoya, 464-8602, Japan
}

We investigate, using a chiral effective model, the quark spectrum in the critical region of the chiral transition focusing on the effect of the possible mesonic excitations in the quark-gluon plasma phase. We find that there appears a novel three-peak structure in the quark spectra. We elucidate the mechanism of the appearance of the multi-peak structure with the help of a Yukawa model with an elementary boson.

\section{Introduction}

It is an intriguing problem with a rather long history to explore the possible existence of hadronic excitations in the quark-gluon plasma (QGP) phase[1, 2], 3]. The existence of the hadronic (mesonic) excitations in the light-quark sector was first predicted as being the soft mode associated to the chiral transition[1]. The recent lattice QCD simulations also suggest the existence of the mesonic bound states in heavy-quark sector in the QGP phase[ [3].

In this work, we investigate the effect of these bosonic excitations on the quark quasiparticle picture in the QGP phase[ 4, 5]. We first explore how the soft mode of the chiral transition[1] affects the quark spectrum in the QGP phase using the Nambu-JonaLasinio(NJL) model, and show that a novel three-peak structure is formed in the quark spectrum near the critical temperature $T_{c}[4]$. We shall elucidate the mechanism and show the universality of the emergence of the three-peak structure in the fermion spectral function, employing a Yukawa model composed of a massless fermion and a massive boson[ 5]: We show that the complicated spectra originate from the mixing between a quark (anti-quark) and an anti-quark hole (quark hole) caused by a "resonant scattering" of the quasi-fermions with the thermally-excited massive boson.

\section{Quark spectrum in NJL model}

To explore the quark matter near $T_{c}$, we first employ the two-flavor Nambu-JonaLasinio (NJL) model in the chiral limit

$\mathcal{L}=\bar{\psi} i \not \partial \psi+G_{S}\left[(\bar{\psi} \psi)^{2}+\left(\bar{\psi} i \gamma_{5} \vec{\tau} \psi\right)^{2}\right]$ 


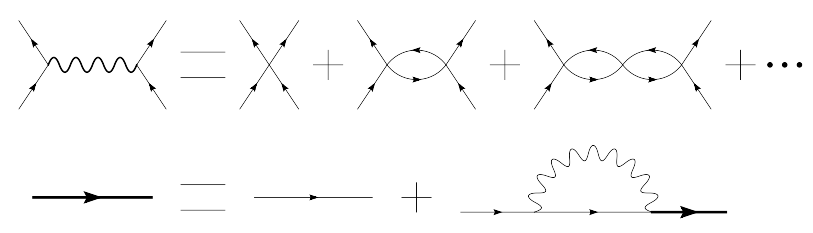

Figure 1. The quark propagator in the RPA. The wavy line denotes the soft modes of the chiral transition.

with the coupling constant $G_{S}=5.5 \mathrm{GeV}^{-2}$ and the three dimensional cutoff $\Lambda=631$ $\mathrm{MeV}$. This model gives a second order phase transition at $T_{c}=193.5 \mathrm{MeV}$ for vanishing quark chemical potential, and the soft modes of the phase transition are dynamically formed in the scalar and pseudoscalar channels. The spectral function in these channels shows a pronounced peak in the time-like region, the peak position and the width of which become smaller and vanishes eventually, as $T$ approaches $T_{c}$ [1, 4].

Now notice that the soft modes composed of quarks can in turn contribute to the quark self-energy $\Sigma$, which may be given in the random phase approximation (RPA) as shown diagrammatically in Fig. 10. In the imaginary time formalism it reads,

$\tilde{\Sigma}\left(\boldsymbol{p}, \omega_{n}\right)=T \sum_{m} \int \frac{d^{3} q}{(2 \pi)^{3}}\left(\mathcal{D}_{\sigma}\left(\boldsymbol{q}, \nu_{m}\right)+3 \mathcal{D}_{\pi}\left(\boldsymbol{q}, \nu_{m}\right)\right) \mathcal{G}_{0}\left(\boldsymbol{p}-\boldsymbol{q}, \omega_{n}-\nu_{m}\right)$,

where $\mathcal{D}_{\sigma, \pi}\left(\boldsymbol{p}, \nu_{n}\right)$ are the Matsubara propagators of the scalar and pseudo-scalar channels and $\mathcal{G}_{0}\left(\boldsymbol{p}, \omega_{n}\right)$ the free quark propagator.

The spectral functions of the quark and anti-quark are expressed as $\rho_{ \pm}(\boldsymbol{p}, \omega) \equiv-(1 / \pi)$ $\operatorname{Tr}\left[\operatorname{Im} G^{R}(\boldsymbol{p}, \omega) \gamma^{0} \Lambda_{ \pm}(\boldsymbol{p})\right]$, with the retarded Green function of the quark $G^{R}(\boldsymbol{p}, \omega)$ and the projection operators $\Lambda_{ \pm}(\boldsymbol{q})=\left(1 \pm \gamma^{0} \boldsymbol{\gamma} \cdot \boldsymbol{q} /|\boldsymbol{q}|\right) / 2$. In the left panel of Fig. 2. we show the quark spectral function $\rho_{+}(\boldsymbol{p}, \omega)$ for $\varepsilon \equiv\left(T-T_{c}\right) / T_{c}=0.1$, i.e., slightly above $T_{c}$. One sees a three-peak structure in the spectral function. Our numerical calculation shows that the clear three-peak structure survives up to $\epsilon \simeq 0.2[4$.

\section{Quark spectrum in Yukawa models}

We have seen that "elementary" bosonic excitations with a small width significantly modify the quark spectrum at finite temperature. To elucidate the mechanism of the modification of the fermion spectra on the more general ground, we employ the Yukawa model composed of a massless quark field $\psi$ and an elementary massive scalar boson $\phi$;

$\mathcal{L}=\bar{\psi}(i \not \partial \psi-g \phi) \psi+\frac{1}{2}\left(\partial_{\mu} \phi \partial^{\mu} \phi-m_{B}^{2} \phi^{2}\right)$.

In this model, the quark self-energy in the imaginary time formalism at the one-loop order is expressed as

$\tilde{\Sigma}\left(\boldsymbol{p}, i \omega_{m}\right)=-g^{2} T \sum_{n} \int \frac{d^{3} \boldsymbol{k}}{(2 \pi)^{3}} \mathcal{G}_{0}\left(\boldsymbol{k}, i \omega_{n}\right) \mathcal{D}\left(\boldsymbol{p}-\boldsymbol{k}, i \omega_{m}-i \omega_{n}\right)$ 

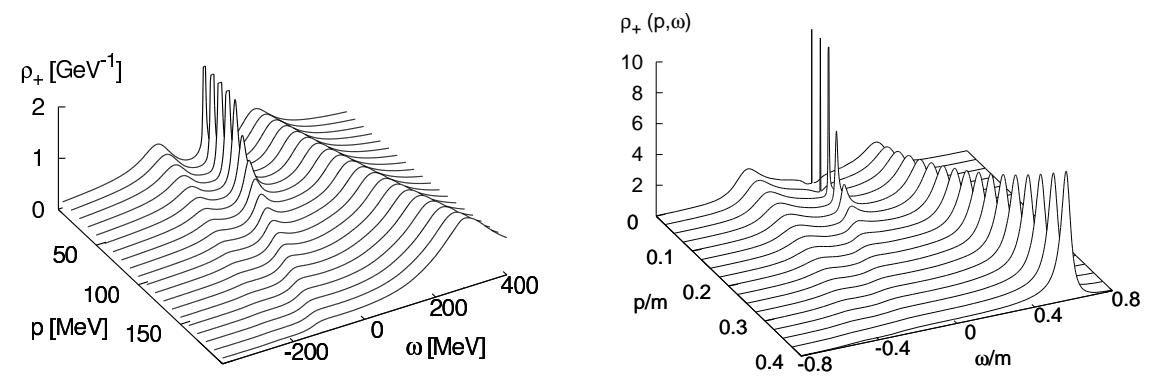

Figure 2. The quark spectral function $\rho_{+}(\boldsymbol{p}, \omega)$ in the NJL and Yukawa models. The left and right panels show $\rho_{+}(\boldsymbol{p}, \omega)$ in the NJL model for $\varepsilon \equiv\left(T-T_{c}\right) / T_{c}=0.1[4]$, and in the Yukawa model for $T / m_{B}=1.4$ [ [5], respectively. There appears the three-peak structure in both models.

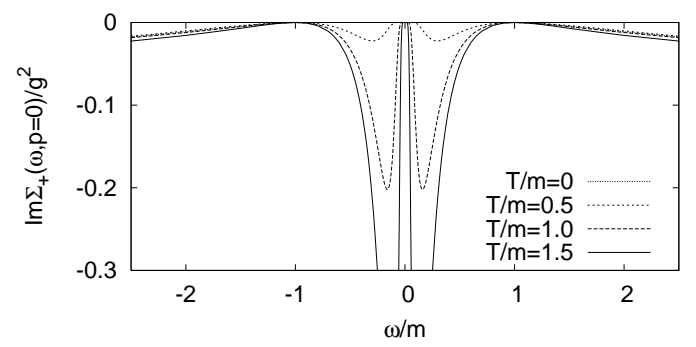

(a)

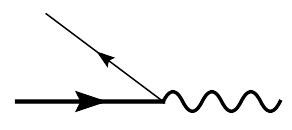

(b)

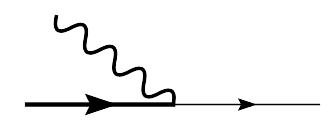

Figure 3. Left panel: The imaginary part of the quark self-energy $\operatorname{Im} \Sigma_{+}^{R}(\mathbf{0}, \omega)$ in the Yukawa model for several values of $T . m$ is the mass of the scalar boson. Right figures: Parts of the physical processes which $\operatorname{Im} \Sigma_{+}^{R}$ describes. The thick lines represent the quasi-quarks, the thin lines the on-shell free quarks and the wavy lines the scalar boson.

where $\mathcal{D}\left(\boldsymbol{k}, i \nu_{n}\right)=\left[\left(i \nu_{n}\right)^{2}-\boldsymbol{k}^{2}-m_{B}^{2}\right]^{-1}$ is the Matsubara Green function for the scalar boson. Taking the Matsubara summation and the analytic continuation, we obtain the self-energy in the real time.

In the right panel of Fig. 2, we show $\rho_{ \pm}(\boldsymbol{p}, \omega)$ at $T=1.4 m_{B}$ and $g=1$. One sees a clear three-peak structure in the spectral function at intermediate temperatures around $m_{B}<T<2 m_{B}[5]$, as in the NJL model.

\section{Discussions}

In order to understand the mechanism of the novel structure in $\rho_{ \pm}(\boldsymbol{p}, \omega)$, we show the imaginary part of $\Sigma_{+}^{R}(\boldsymbol{p}=0, \omega)$ in the Yukawa model with several $T$ in the left panel in Fig. 3. There are two peaks in $\left|\operatorname{Im} \Sigma_{+}^{R}\right|$ with a positive and negative energy, which grow rapidly as $T$ is raised. It can be easily checked that these peaks in the positive and negative energy regions physically correspond to the Landau dampings shown in the right panel in Fig. 3(a) and (b), respectively; the wavy line represents the scalar boson. We remark that the incident anti-quark line in Fig. 3(a) describes a thermally excited antiquark, 
which disappears after the collision with the scalar boson. The disappearance of the anti-quark means the creation of a hole in the thermally-excited anti-quark distribution[ 6]. Figure 3(b) describes the decay process of a quasi-quark which is a mixed state of quarks and antiquark-holes to an on-shell quark. The two processes induce a respective quark-'antiquark hole' mixing and hence two gaps are realized in $\rho_{ \pm}$at $\omega$ where $\operatorname{Im} \Sigma_{ \pm}^{R}$ has a peak corresponding to each process.

The mixing mechanism of the quarks can be understood in terms of the notion of resonant scattering as in the case of the (color-)superconductivity [ 7, 8], although a crucial difference arises owing to the different nature of the bosonic modes. In the case of the superconductivity, the precursory soft mode which induces the mixing is diffusionmode like and has a strength around $\omega=0[9]$. The resonant scattering with the soft mode induces the mixing between a particle and a hole, and gives rise to a gap-like structure in the fermion spectrum around the Fermi energy[ [10, 8]; correspondingly, the imaginary part $\left|\operatorname{Im} \Sigma^{R}\right|$ of the quark self-energy has a single peak around the Fermi energy[ [8]. In the present cases, the bosonic modes have a strength at finite energies and hence the resonant scattering of the quarks bring the two peaks in $\left|\operatorname{Im} \Sigma^{R}\right|$ at finite energies and induces a mixing between a quark (antiquark) and an antiquark-hole (quark-hole). Thus the two gaps in the quark spectrum are formed in the positive- and negative-energy regions.

In summary, using the NJL model, we have shown that the quark spectrum in the QGP phase near the critical temperature of the chiral transition can have a three-peak structure in the low-momentum region owing to the effect of the hadronic soft modes of the chiral transition. It has been shown that the three-peak structure in the fermion spectra also arises in the Yukawa model with an elementary massive boson. The detailed analysis of this model have elucidated the mechanism for the appearance the three-peak structure, which is realized as a result of the mixing between a quark(anti-quark) and antiquark-hole (quark-hole) induced by the resonant scattering of the quarks.

T.K. is supported by Grant-in-Aid for Scientific Research by Monbu-Kagakusyo (No. 17540250). Y.N. is supported by the 21st Century COE progaram of Nagoya University and Grant-in-Aid for Scientific Research by Monbu-Kagakusyo (No. 18740140).

\section{REFERENCES}

1. T. Hatsuda and T. Kunihiro, Phys. Lett. B145, 7 (1984); Phys. Rev. Lett. 55, 158 (1985).

2. T. Matsui and H. Satz, Phys. Lett. B 178, 416 (1986).

3. M. Asakawa and T. Hatsuda, Phys. Rev. Lett. 92, 012001 (2004); S. Datta, et al., Phys. Rev. D 69, 094507 (2004); T. Umeda, et al., Eur. Phys. J. C 39S1, 9 (2005).

4. M. Kitazawa, T. Kunihiro and Y. Nemoto, Phys. Lett. B633, 269 (2006).

5. M. Kitazawa, T. Kunihiro and Y. Nemoto, in preparation.

6. H. A. Weldon, Phys. Rev. D 61, 036003 (2000).

7. B. Jankó, J. Maly and K. Levin, Phys. Rev. B 56, R11407 (1997).

8. M. Kitazawa, T. Kunihiro and Y. Nemoto, Phys. Lett. B631, 157 (2005).

9. M. Kitazawa, T. Koide, T. Kunihiro and Y. Nemoto, Phys. Rev. D 65, 091504 (2002); Prog. Theor. Phys. 114, 205 (2005).

10. M. Kitazawa, T. Koide, T. Kunihiro and Y. Nemoto, Phys. Rev. D 70, 056003 (2004). 